\title{
Advances in Microscopy of Semiconducting Materials Featured at 6 th International Conference
}

The 6th International Conference on Microscopy of Semiconducting Materials concentrated on the important applications of, primarily, electron microscopy and related analytical techniques to the study of the structure and properties of semiconductors. The latest in a biennial series, the conference took place at the University of Oxford, United Kingdom, April $10-13,1989$ and attracted delegates from 20 countries.

Conference sponsorship was provided by the Royal Microscopical Society, the Institute of Physics (UK), and the Materials Research Society. The meeting was chaired by Tony Cullis (RSRE, Malvern) and John Hutchison (Oxford University). All major topic areas from basic research to device development were covered by the 131 scientific papers, half presented orally and half displayed as posters. Leading experts introduced individual topics with keynote invited papers.

The meeting was opened by Sir Peter Hirsch (1983 MRS Von Hippel Award recipient), and the first session focused on applications of high resolution transmission electron microscopy. J. Van Landuyt (Antwerp University) demonstrated how lattice and atomic imaging could be used to characterize the finest details of ionimplanted silicon. Topics ranged from defect structures in low-dose implanted silicon to phase formation after high-dose implantation. The session continued with papers describing advances in our understanding of compound semiconductor lattice images, the local atomic composition at interfaces and the core structure of dislocations. Impressive pictures were presented showing the atomic structure of semiconductor interfaces obtained by direct atomic-number-contrast imaging in the scanning transmission electron microscope.

Advances in the study of processed silicon were covered in two other sessions. The latest techniques designed to yield atomically thin specimens of multi-component device structures for transmission microscopy were described by R.M. Anderson (IBM, Hopewell Junction). Elegant studies of gold/silicon interfaces and oxygen precipitation in silicon were presented by R.W. Carpenter (Arizona State University), who also illustrated the use of electron energy loss spectroscopy for chemical analysis of extremely small specimen regions. Other papers emphasized dopant profiling in silicon device structures using novel selective etch-

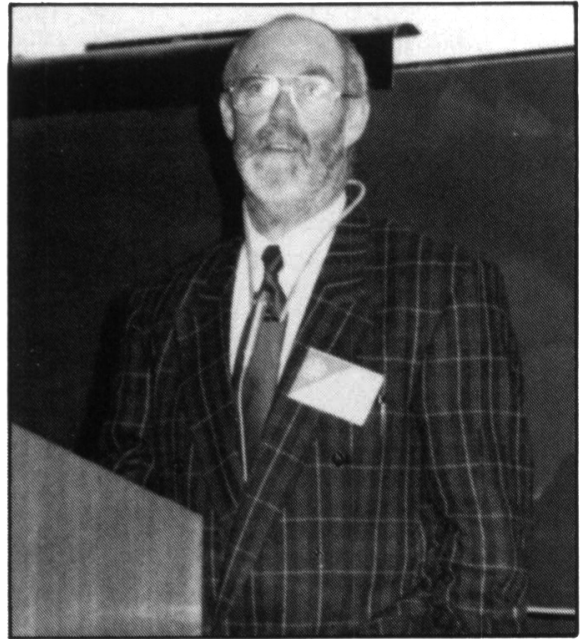

J. Van Landuyt surveys advances in high resolution microscopy applications.

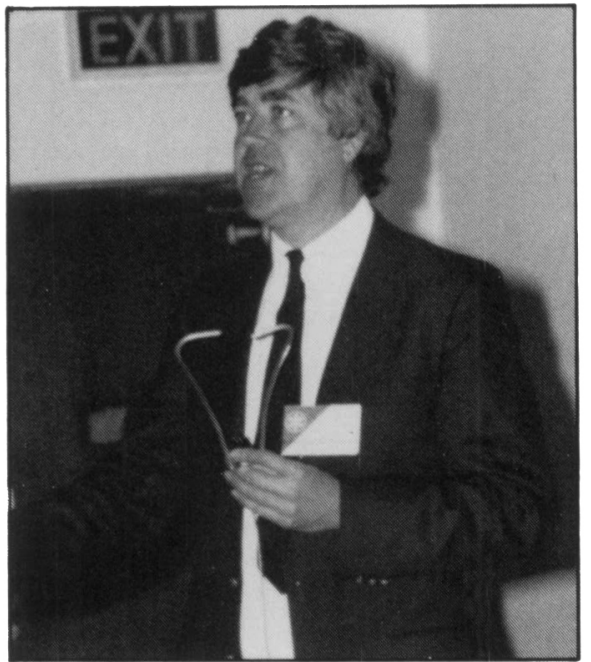

R. Sinclair introduces dynamic studies of metal-silicon reactions and silicon regrouth.

ing procedures followed by microscopic analysis. The cross-sectional characterization of silicon-on-insulator material formed by oxygen and nitrogen ion implantation was also featured.

Work on metal semiconductor contacts and silicides was of particular interest. R. Sinclair (Stanford University) presented fascinating, high resolution, dynamic studies of silicide forming reactions at metal-silicon interfaces. Overall, there was detailed consideration of the formation of disilicides such as those of cobalt and titanium in relation to IC fabrication. The nature of cobaltgallium and nickel metallization on gallium arsenide was also explored in detail.

Advances in the important area of electron-beam testing of submicron devices were reviewed by $\mathrm{E}$. Wolfgang (Siemens Laboratories, Munich). This topic was extended in additional papers which considered applications of image processing for $\mathrm{IC}$ characterization and the effects of electron beam irradiation on transistor properties.

Fundamental research on epitaxial layer systems was represented especially strongly at the conference. The study of the initial growth of silicon layers using the important phenomenon of RHEED oscillations was described in depth by T. Sakamoto (Electrotechnical Laboratory, Ibaraki). Accompanying papers covered areas ranging from the growth of gallium arsenide and silicon carbide on silicon to phase transformation in $\alpha$-tin films on indium antimonide. Recent developments in the understanding of atomic ordering and phase separation in ternary and quaternary III-V alloys were summarized by S. Mahajan (Carnegie Mellon University, Pittsburgh). Other workers presented a range of perspectives on the ordering processes. D.M. Maher (AT\&T Bell Laboratories, Murray Hill) extended the topic to structure/property considerations of device quality heterojunctions; discussion of state-of-the-art work on dislocation engineering in III-V device structures followed.

Work on superlattices and quantum wells aroused considerable interest. Advances in microscopy techniques used to characterize these structures were reviewed in some detail by W.M. Stobbs (Cambridge University). This was followed by several studies of well structure and sharpness by exploitation of both conventional and high resolution transmission electron microscopy. Studies of superlattices and epitaxial layers by $x$-ray topography and diffractometry were strongly represented: the present status of their application to the characterization of cadmium mercury telluride was comprehensively covered by G.T. Brown (RSRE, Malvern).

A special session was devoted to the latest developments in bulk gallium arsenide research. G.M. Martin (LEP, LimeilBrévannes) summarized the current status of work on precipitation phenomena in the 
bulk material and their relationships to MESFET IC yield. Further presentations extended this topic with detailed correlations involving important point defect characteristics. The distinctive behavior of dislocation arrays was also featured, particularly concerning the effects of indium doping.

The development and exploitation of advanced microscopy techniques was a special feature of the conference. N.J. Long (Oxford University) described recent applications of $\mathrm{x}$-ray microanalysis in the scanning transmission electron microscope to determine the local composition of compound semiconductor alloy systems. Special emphasis was placed on the effects of electron channeling as a function of crystallographic orientation. Subsequent presentation of related work on the silicon/germanium system was followed by description of the impressive atomic resolution capabilities of atom probe microanalysis. Developments in the quantitative characterization of semiconductors by electron-beam-induced conductivity were brought to a focus in the review by $C$. Donolato (LAMEL, Bologna). Other papers in the field highlighted advances in the elucidation of the electrical properties of defects by use of the latter technique applied to dislocation and grain boundary studies in silicon, together with cathodoluminescence which was employed to characterize compound semiconductors including heterostructures. Novel structural and electrical measurements made using scanning optical microscopy where also featured.

Thin specimen preparation for transmission electron microscopy and elemental microanalysis were the subjects of evening workshops, which attracted large numbers of participants for lively exchanges of information. In addition, B.A. Joyce (Imperial College, London) presented the annual RMS materials lecture one evening. His topic, "Tailor-Made Semiconductor Structures with Atomic Dimensions-Band Structure Engineering," struck exactly the right note and was extremely well received by the packed audience.

Overall, the conference, which had been extended to four days, kept delegates very busy. The proceedings volume is due to be published toward the end of 1989 . It will be available from (United States and Canada) American Institute of Physics, c/o AIDC, 64 Depot Road, Colchester, VT 05446, telephone (800) 445-6638); or (elsewhere) IOP Publishing Ltd., Techno House, Redcliffe Way, Bristol BS16NX, UK, telephone 0272297481.

Anthony G. Cullis

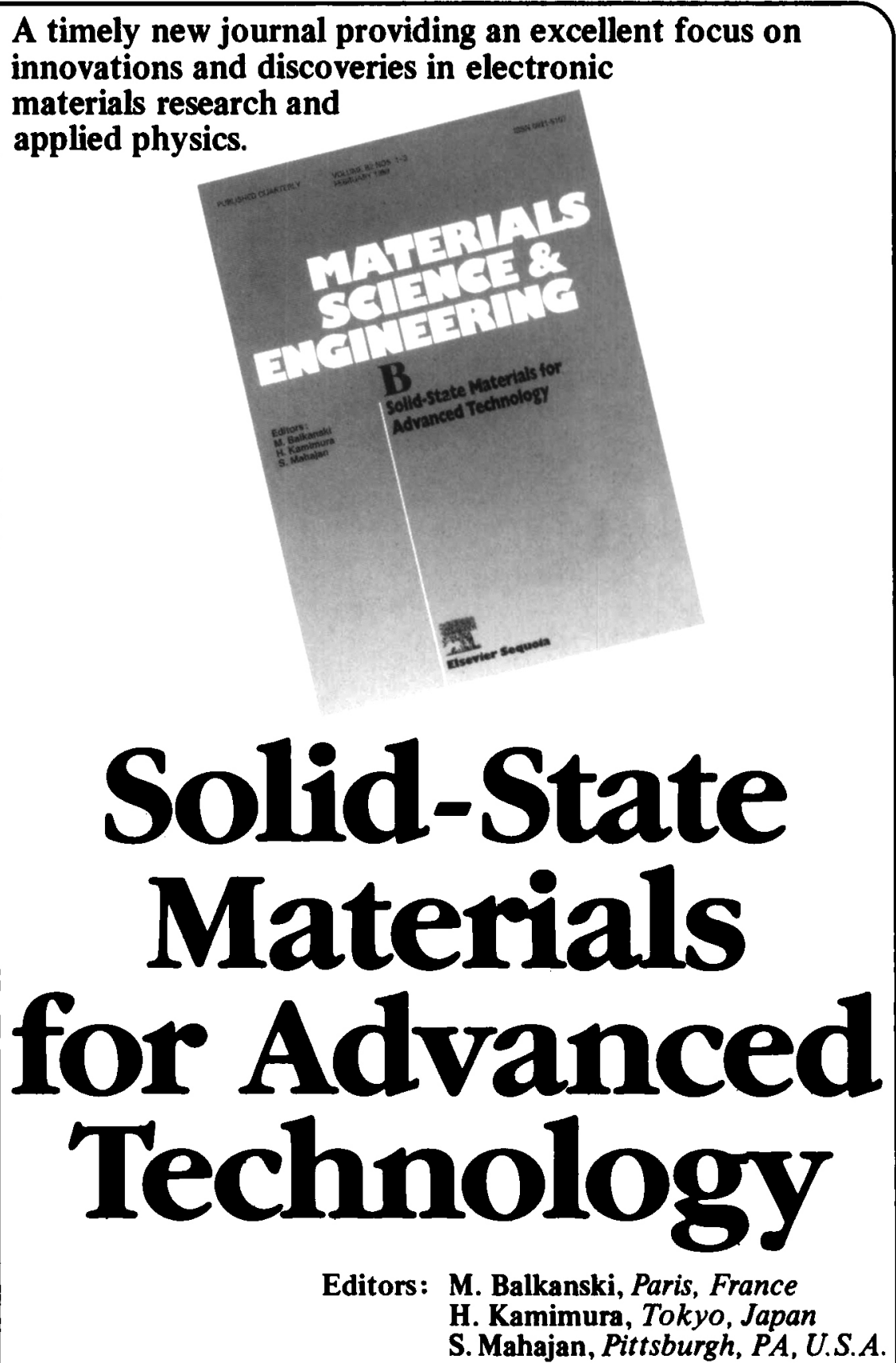

This international journal covers the full range of topics pertaining to the expanding field of solid-state physics and materials science of electronic materials.

The Journal covers processing, characterization and structure of materials both in bulk and in thin films.

\section{Materials include: \\ - Semiconductors \\ - Superconductors \\ - Low-Dimensional Compounds \\ - Magnetic and Optoelectronic Materials \\ - Fast ion conductors}

Ask for a FREE sample copy

Elsevier Sequoia SA

P.O. Box 564

1001 Lausanne 1, Switzerland

AMSTERDAM - LAUSANNE

LONDON - NEW YORK - OXFORD - TOKYO

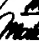

ELSEVIER 